\title{
Optical and rheological studies on weak gel-sol transition in aqueous solutions of $\operatorname{poly}(N$-isopropylacrylamide)-block-polystyrene
}

\author{
S. Sanjeevi Prasath ${ }^{1}$, J. Brijitta ${ }^{*}$, B. V. R. Tata ${ }^{2}$, R. G. Joshi ${ }^{3}$, K. Chennakesavulu ${ }^{4}$, Deepak K. Gupta ${ }^{3}$ \\ ${ }^{1}$ Centre for Nanoscience and Nanotechnology, Sathyabama University, Chennai-600119, India \\ ${ }^{2}$ Department of Physics, Indian Institute of Technology Palakkad, Palakkad-678557, India \\ ${ }^{3}$ Condensed Matter Physics Division, Materials Science Group, Indira Gandhi Centre for Atomic Research, \\ Kalpakkam-603102, India \\ ${ }^{4}$ Department of Chemistry, Sathyabama University, Chennai-600119, India
}

Received 23 November 2016; accepted in revised form 21 February 2017

\begin{abstract}
The optical and rheological properties of aqueous solutions of block copolymer composed of low molecular weight poly( $N$-isopropylacrylamide)- $b$-polystyrene are studied as a function of temperature. From light scattering measurements the block copolymer solution is found to form micelles at very low concentrations and the critical micellar concentration is identified as $0.005 \mathrm{wt} \%$. Apart from the concentration dependence, a unique temperature dependent micelle formation is noted at $34^{\circ} \mathrm{C}$. Further, temperature dependent refractive index measurements shows that the refractive index increases with temperature (beyond the lower critical solution temperature, $31.6^{\circ} \mathrm{C}$ of the polymer), and is attributed to the stable rearrangement of the proximal hydrophobic isopropyl-polystyrene chains in the collapsed polymer so as to overcome the steric hindrance effects offered by the hydrophobic chains. In the polymer concentrations investigated for rheological studies, the solution flows, yet manifested solid like behavior with $G^{\prime}>G^{\prime \prime}$ with the modulus being frequency dependent and the magnitude of $G^{\prime}$ two-fold higher than $G^{\prime \prime}$ implying a weak gel state. Weak gel states are in general noted at high temperatures in most of the polymer systems, contrary to this, in our studies weak gel state is observed at lower temperature. Further, a transition from weak gel to sol state is observed at slightly elevated temperatures. The reason for the existence of weak gel state below the lower critical solution temperature is due to the micellar entanglements of poly( $N$-isopropylacrylamide)- $b$-polystyrene with one another and whereas above the lower critical solution temperature disentanglement of the micelles makes the system behave like a viscoelastic liquid.
\end{abstract}

Keywords: rheology, block copolymer, PNIPAM, light scattering, micelles

\section{Introduction}

With the recent developments in synthesis strategies of novel macromolecules, it is now possible to tune the macromolecular size and shape by covalent and non-covalent interactions. The covalent interactions can be effectively tapped to form block copolymers (BCPs) which has numerous applications such as, an etch mask for nano/meso scale patterning, photovoltaic devices, drug delivery, etc [1-6]. Apart from the cited applications, BCPs are also being explored for their applicability as photonic materials [7-12]. Among the numerous strategies of controlled/living free-radical polymerization for the synthesis of $\mathrm{BCPs}$, reversible addition-fragmentation chain transfer (RAFT) polymerization process stays in the forefront. The advantage of RAFT polymerization is that, it results in polymers with controlled molecular weight and with narrow polydispersities and offers a wide 
range of monomer selection and reaction conditions. The mechanism of RAFT polymerization occurs through a reversible addition-fragmentation sequence in which the transfer agent exhibits very high transfer constants for a rapid rate of exchange between dormant and living chains. Some of the routinely employed RAFT chain transfer agents (CTA) are trithiocarbonates [13-15], dithiocarbamates [1618], dithiobenzoates [19-22], etc.

Imparting responsive nature to the block copolymers enables to understand the behavior of the polymer (shape, permeability, or color) in response to a series of changes in external stimuli such as $\mathrm{pH}$, temperature, salt concentration, charge, light, etc. Despite the synthesis challenges, responsive block copolymers can produce well-defined materials either by triggered or non-covalent associations of one or more blocks. This in turn imparts the tunability of the physical properties of the polymer such as colour, shape, Young's modulus of elasticity, tacticity, glass transition temperature, etc, subjected to changes in stimuli. Polymers based on poly( $N$-isopropylacrylamide) (PNIPAM) and poly( $N$-vinylcaprolactam) (PVCL) exhibit thermo-sensitivity, and those based on poly (acrylic acid) (PAA) and poly(methacrylic acid) (PMAAc) exhibit pH sensitivity. By making use of amphiphilic diblock copolymers, it is possible to achieve self-assembly of the block copolymer segments into spherical micelles by selectively solubilizing only one block in a suitable solvent [23]. The micelles can be disrupted by applying stimulus such as $\mathrm{pH}$, temperature, solvent/salt concentration. In a study by Tang et al. [24], the temperature, $\mathrm{pH}$, and sugar induced micellization and dissociation transition of poly(3-acrylamidophenylboronic acid)- $b$ poly( $N$-isopropylacrylamide) (PAAPBA- $b$-PNIPAM) block copolymer was investigated by light scattering technique and the block copolymer self-assembles into micelles with the core made up of PAAPBA and shell of PNIPAM. An increase in temperature led the micelles to shrink at $\mathrm{pH}$ 6.2, whereas at an elevated $\mathrm{pH}$ of 10.0 , due to the increase in hydrophilicity of the PAAPBA block, the micelles completely dissociated to unimers and proceeds through a step-bystep sequential expulsion of individual chains. Poggi et al. [25] showed that, the self-assembly properties of poly(vinyl alcohol)-block-poly(acrylic acid)block-poly(4-vinylpyridine) triblock terpolymer (PVOH-b-PAA- $b$-P4VP) in water as a function of $\mathrm{pH}$ exhibited three different aggregation states and the micellar disassembly can be achieved by changing the temperature. The aggregation number of the PVOH- $b$-PAA- $b$-P4VP micelles and their size in turn can be tailored by controlling the ionic strength of the solution prior to micellization. Further the charged PAA segments in the shell upon the addition of calcium ions collapses onto the P4VP core. Since our group has a long-standing interest in stimuli responsive polymers based on PNIPAM, which exhibits a lower critical solution temperature (LCST) at $\sim 32{ }^{\circ} \mathrm{C}$ [26-30], we too synthesized low molecular weight hydrophilic-hydrophobic block copolymers of poly( $N$-isopropylacrylamide)-block-polystyrene (PNIPAM- $b-\mathrm{PS}$ ) by RAFT polymerization using 2(dodecylthiocarbonothioylthio)-2-methylpropionicacid (DDMAT).

The PNIPAM- $b$-PS block copolymer upon selective solubilization of the PNIPAM segments can form micelles in water; and thus it will be of interest to investigate the effect of temperature on the micellar behaviour. The effect of temperature and concentration on aqueous solutions of the PNIPAM- $b$-PS block copolymer optical and rheological properties is reported in this paper. The micellar behaviour (size change) can be studied using dynamic light scattering technique. It is observed that micelle formation is not just concentration dependent but also depends on the temperature. The rheological studies on aqueous solutions of the block copolymer reveal a weak gel to sol transition across the LCST different from the existing reports [31-33]. In the polymer concentrations investigated, the solution flows, yet manifested solid like behavior with $G^{\prime}>G^{\prime \prime}$ with the modulus being frequency dependent and the magnitude of $G^{\prime}$ is two-fold higher than $G^{\prime \prime}$ which implies a weak gel state. Weak gel states are in general noted at high temperatures in other polymer systems, contrary to this, in our studies weak gel state is observed at lower temperature. A plausible reason for the weak gel behavior is discussed. Apart from the concentration dependence, it is observed that temperature too plays a crucial role in the formation of micelles beyond the LCST of the block copolymer.

\section{Experimental \\ 2.1. Chemicals}

$\mathrm{N}$-isopropylacrylamide (NIPAM, 97\%), styrene containing 4-tert-butylcatechol (99\%), 2, 2-azobis (2methylpropionitrile) (AIBN, 98\%), 1, 4-dioxane (99.8\%) and 2-(dodecylthiocarbonothioylthio)-2- 
methylpropanoic acid (DDMAT) (98\%) were purchased from Sigma Aldrich. NIPAM was recrystallized in hexane. Styrene was washed with $\mathrm{NaOH}$ to remove the stabilizer. All the chemicals used were of analytical grade and used as received. The glass wares were pre-cleaned and dried before use.

\subsection{Synthesis of macro}

\section{$\operatorname{poly}(N$-isopropylacrylamide) -chain} transfer agent (macro PNIPAM-CTA)

NIPAM (0.8 mol), DDMAT (0.2 mmol) and AIBN (1.6 mmol), was added to $80 \mathrm{~mL}$ of 1 , 4-dioxane in a round-bottom flask equipped with a stir bar. The reaction flask was sealed with a rubber septum and purged with nitrogen gas for $30 \mathrm{~min}$ in an ice bath. The solution was degassed by three freeze-pump-thaw cycles followed by quick vacuum sealing and placing it in a thermostatically controlled oil bath and the reaction was allowed to proceed at $70^{\circ} \mathrm{C}$ for $60 \mathrm{hrs}$. After the polymerization the reaction mixture was cooled to room temperature and precipitated by adding cold ether. The resultant macro PNIPAMCTA had a pale yellow tinge and the yield was quantified as $5.8 \mathrm{~g}$.

\subsection{Synthesis of \\ poly( $N$-isopropylacrylamide-lock-styrene) (PNIPAM-b-styrene)}

For the synthesis of PNIPAM- $b$-styrene from macro PNIPAM-CTA, $2.50 \mathrm{~g}$ of macro PNIPAM-CTA, styrene $(0.4 \mathrm{~mol})$ and AIBN (1.54 mmol) was added to $50 \mathrm{~mL}$ of 1, 4-dioxane. After sealing the reaction flask, nitrogen gas was purged for $30 \mathrm{~min}$ and the solution was degassed by three freeze-pump-thaw cycles following which the reaction flask was vacuum sealed and reacted at $80^{\circ} \mathrm{C}$ for $24 \mathrm{hrs}$. After the polymerization the reaction mixture was cooled and precipitated from cold ether, yielding $2.3 \mathrm{~g}$ of yellow solids. Figure 1 summarizes the synthetic route for obtaining the diblock copolymer PNIPAM- $b$-PS from its monomer and CTA.

\section{Characterization techniques}

\subsection{FTIR, NMR and GPC}

Macro PNIPAM-CTA and PNIPAM- $b$-PS block copolymers were characterized by Fourier-transform infrared spectroscopy (FTIR) and ${ }^{1} \mathrm{H}$ nuclear magnetic resonance spectrometry (NMR). FTIR spectra of the polymer was recorded on a Fourier Transform Infrared Spectrophotometer (IR Tracer-100 Shimadzu) using the $\mathrm{KBr}$ pellet technique. From the FTIR spectrum, the presence of PNIPAM-CTA is confirmed by the peaks at $1070-1246 \mathrm{~cm}^{-1}$ (stretching vibration of $\mathrm{C}=\mathrm{S}$ and $-\mathrm{C}-\mathrm{S}-\mathrm{C}-$ ), the peaks at $3292 \mathrm{~cm}^{-1}$ correspond to the secondary amide $\mathrm{N}-\mathrm{H}$ stretching $\mathrm{cm}^{-1}$, the peak at $1640 \mathrm{~cm}^{-1}$ shows secondary amide $\mathrm{C}=\mathrm{O}$ and acid group $(\mathrm{COOH})$ and the $\mathrm{CH}_{3}$ asymmetric stretching vibration bands appear at $2970 \mathrm{~cm}^{-1}$. PNIPAM- $b$-PS was identified by the peaks at $3076 \mathrm{~cm}^{-1}$ (C-H aromatic tension), 615$879 \mathrm{~cm}^{-1}$ (aromatic C-H bending), $1500-1700 \mathrm{~cm}^{-1}$ (aromatic $\mathrm{C}=\mathrm{C}$ bending), $\mathrm{CH}_{2}$ asymmetric and symmetric tension at $2875-2976 \mathrm{~cm}^{-1}$. A Bruker $300 \mathrm{MHz}$ ${ }^{1} \mathrm{HNMR}$ spectrometer was used with $\mathrm{CDCl}_{3}$ as the solvent and tetramethylsilane as the internal reference. The macro PNIPAM-CTA showed chemical shifts in the ${ }^{1} \mathrm{HNMR}$ at $0.99-1.30\left(\mathrm{~m}, 3 \mathrm{H},-\mathrm{CH}_{3}\right)$, $1.70-2.50\left(\mathrm{~m},-\mathrm{CH}_{2}-\mathrm{CH}-\right), 3.55\left(-\mathrm{CH}_{2} \mathrm{~S}\right),(\mathrm{m},-\mathrm{CH}$ $\left(\mathrm{CH}_{3}\right)_{2}$ and $-\mathrm{CH}_{2}-\mathrm{CH}-$ on the polymer's main chain), $4.02\left(\mathrm{~s},-\mathrm{NHCH}\left(\mathrm{CH}_{3}\right)_{2}\right)$ and the chemical shifts for the PNIPAM- $b$-PS block copolymer is $1.16-2.64(\mathrm{~m}$, $-\mathrm{CH}\left(\mathrm{CH}_{3}\right)_{2}$ and $-\mathrm{CH}_{2}-\mathrm{CH}-$ on the polymer's main chain), $4.02\left(\mathrm{~s},-\mathrm{NHCH}\left(\mathrm{CH}_{3}\right)_{2}\right), 5.67-7.0\left(\mathrm{~m}, \mathrm{C}_{6} \mathrm{H}_{5}\right.$ of styrene and $\left.-\mathrm{NHCH}\left(\mathrm{CH}_{3}\right)_{2}\right)$. The molecular weights and the polydispersities index (PDI) of the macro PNIPAM-CTA, and the PNIPAM- $b$-PS block copolymers were determined by gel permeation chromatography (GPC), using an Auto system XL GC with Turbo mass from Perkin Elmer. GPC was performed with tetrahydrofuran as solvent at $45^{\circ} \mathrm{C}$ at a flow rate of $1.0 \mathrm{~mL} / \mathrm{min}$, and the calibration was based on a set of monodisperse polystyrene standards. The GPC curve exhibits unimodal distribution,

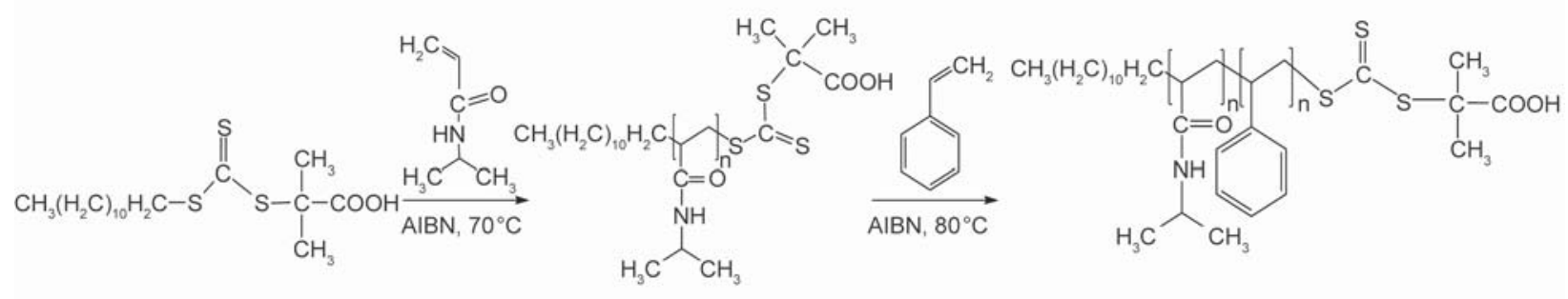

DDMAT

Figure 1. Synthetic route for obtaining the diblock copolymer PNIPAM- $b$-PS 
implying monodisperse sample and homopolymers being absent in the block copolymer. The molecular weight of macro PNIPAM-CTA and PNIPAM- $b$-PS block copolymer is found to be 6894 and $9695 \mathrm{~g} / \mathrm{mol}$ with PDI of 1.25 and 1.27 respectively. The GPC measurements have been repeated and the results were reproduced with an accuracy of $0.1 \%$. There are several reports on block copolymers synthesized by RAFT polymerization exhibiting unimodal and narrow molecular weight distribution [34-37]. From the molecular weight the number of PNIPAM and polystyrene segments is found to be 67 and 27 respectively and hence the short form PNIPAM $_{67}-b-\mathrm{PS}_{27}$ is used in the entire text to denote the block copolymer. In arriving at the number of monomer segments the contribution of the chain transfer agent is neglected.

\subsection{DLS, transmission spectroscopy, refractometry}

The hydrodynamic diameter and effect of temperature on the hydrodynamic diameter of PNIPAM $_{67}-b-$ $\mathrm{PS}_{27}$ block copolymers was determined by Dynamic Light Scattering, using Malvern-UK, 4700 model. In DLS, the intensity-intensity auto correlation function $g^{(2)}(q, t)$, measured at a given scattering wave vector, $q$ is given by Equations (1) and (2):

$$
\begin{aligned}
& g^{(2)}(q, t)=\frac{I(q, 0) I(q, t)}{(I(q, 0))^{2}} \\
& q=\frac{4 \pi \mu_{\text {medium }}}{\lambda} \sin \left(\frac{\theta}{2}\right)
\end{aligned}
$$

where $\mu_{\text {medium }}$ is the refractive index of the medium, $\theta$ is the scattering angle and $\lambda$ is the wavelength of the incident laser light. The normalized electric field autocorrelation function $f(q, t)$ is related to $g^{(2)}(q, t)$ by the Siegert-relation [27] (Equation (3)):

$$
g^{(2)}(q, t)=1+\beta|f(q, t)|^{2}
$$

where $\beta$ is the coherence factor. In a non-interacting system, $f(q, t)$ can be expressed by Equation (4):

$f(q, t)=\exp \left(-D_{0} q^{2} t\right)$

where $D_{0}$ is the free diffusion coefficient and is related to the hydrodynamic diameter, $d_{\mathrm{h}}$ by the StokesEinstein relation given by Equation (5):
$d=\frac{k_{\mathrm{B}} T}{3 \pi \eta D_{0}}$

where $k_{\mathrm{B}}, \eta$ and $T$ are the Boltzmann constant, the solvent (water) viscosity and the absolute temperature, respectively. In the DLS measurements, the Zaverage diameter given as the output of the measurements by the instrument refers to the mean hydrodynamic diameter, $d_{\mathrm{h}}$ and the polydispersity index estimates the width of the distribution.

Temperature dependent transmittance of the PNIPAM $_{67}-b-\mathrm{PS}_{27}$ block copolymers was determined using a UV-Visible spectrophotometer (JASCO V670 , Japan). The refractive indices of the block copolymer solution were measured at various temperature $\left(20-40^{\circ} \mathrm{C}\right)$ using Rudolph Research Analytical, J457 Automatic Refractometer, USA.

\subsection{Rheology}

Rheological measurements on aqueous solutions for various concentrations of PNIPAM $_{67}-b-\mathrm{PS}_{27}$ block copolymer have been carried out using a MCR 301 Rheometer (Anton Paar, Germany) in the double gap geometry. Frequency sweep-measurements have been performed in the range of 0.1 to $100 \mathrm{rad} / \mathrm{s}$ to measure $G^{\prime}(\omega)$ and $G^{\prime \prime}(\omega)$, the linear viscoelastic storage modulus and the loss modulus respectively.

\section{Results and discussion \\ 4.1. Effect of temperature on the optical properties}

The critical micellar concentration (CMC) of the PNIPAM $_{67}-b$ - PS $_{27}$ block copolymer has been determined by DLS technique, by preparing a series of aqueous solutions in the concentration range of 0.0001 to $0.05 \mathrm{wt} \%$ at $25^{\circ} \mathrm{C}$. The intensity of scattered light as a function of concentration is shown in Figure 2. The intercept of the two slopes is identified as the CMC of the polymer which is $0.005 \mathrm{wt} \%$. Below the CMC almost constant scattering intensities is detected whereas beyond the CMC the scattered light intensity profile showed a linear increase with concentration.

Having established the CMC of the PNIPAM P7- $_{6-}$ $\mathrm{PS}_{27}$ block copolymer solution as $0.005 \mathrm{wt} \%$, the hydrodynamic size of the micelles and the effect of temperature on the size of the micelles is determined by DLS. At $25^{\circ} \mathrm{C}$, the hydrodynamic diameter of the 


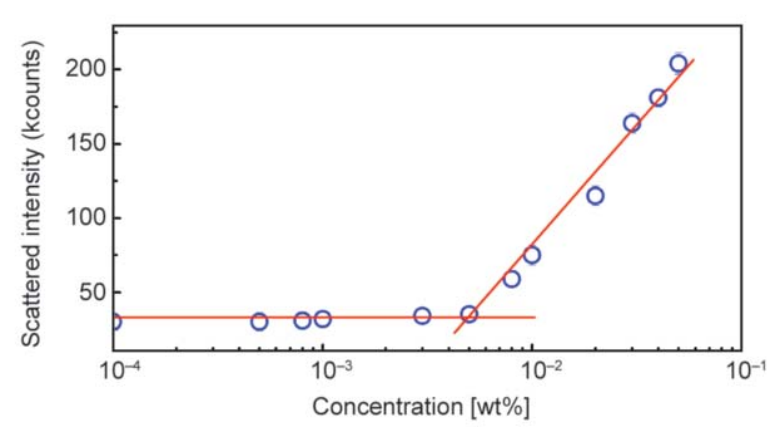

Figure 2. Scattered intensity as a function of concentration of PNIPAM P $_{67}-b-\mathrm{PS}_{27}$ at $25^{\circ} \mathrm{C}$

micelles is found to be $85.2 \pm 5.66 \mathrm{~nm}$. Figure 3 shows the plot of hydrodynamic diameter, $\mathrm{d}$ of the micelles as a function of temperature for the $0.005 \mathrm{wt} \%$ PNIPAM $_{67}-b-\mathrm{PS}_{27}$ block copolymer solution. The temperature induced collapse of the PNIPAM $_{67}-b-$ $\mathrm{PS}_{27}$ aggregates is observed at $31.6^{\circ} \mathrm{C}$ and the collapse can be explained as follows. Upon contact with water the amide side chains of PNIPAM block form intermolecular hydrogen bonding with the surrounding water molecules. It is also reported that there is also some structured water around the isopropyl groups of PNIPAM [38]. This results in strong polymer-water (solvent) interaction and as a consequence the polymer swells in the solvent. Beyond a certain temperature known as the lower critical solution temperature (LCST) the inter-molecular hydrogen bonds with the water molecules break. The characteristic LCST of the PNIPAM is $\sim 32-33^{\circ} \mathrm{C}$ at which the PNIPAM undergoes an entropically favored volume change (breaking of the hydrogen bonds) [26$28,38-41]$, the LCST range is tunable by the addition of co-monomers leading to the deswelling of the polymer chains. Beyond the LCST of the polymer, the turbidity of the solution intensifies. This change in

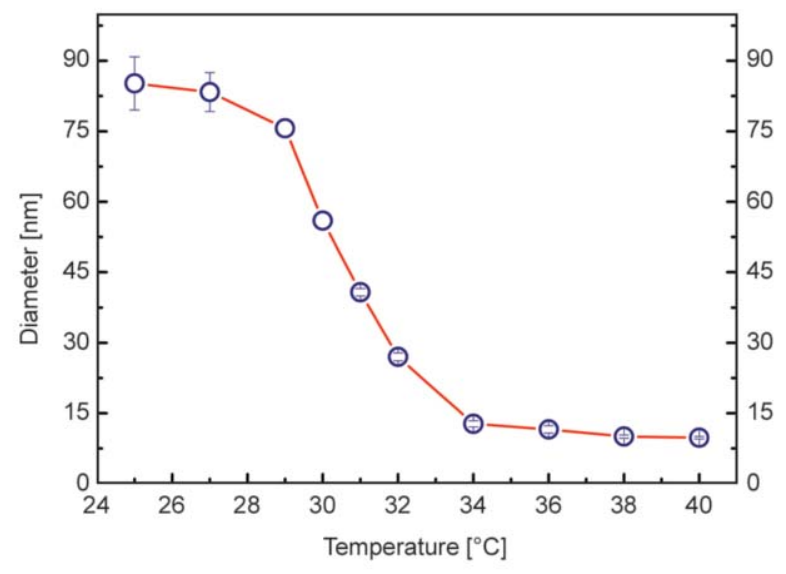

Figure 3. Temperature dependence of hydrodynamic diameter $\left(d_{\mathrm{h}}\right)$ of PNIPAM $_{67}-b-\mathrm{PS}_{27} 0.005 \mathrm{wt} \%$ aqueous solution

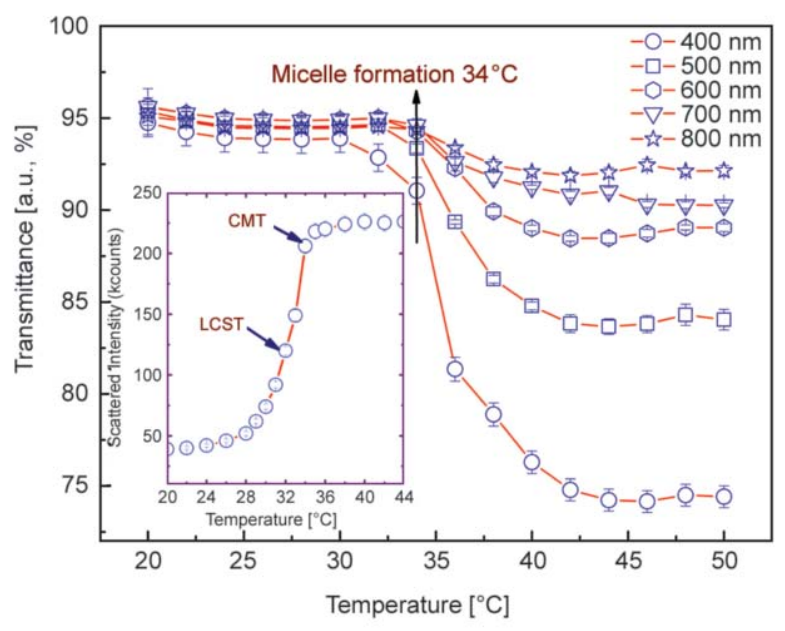

Figure 4. Transmittance change as a function of temperature for $0.001 \mathrm{wt} \%$ PNIPAM $_{67}-b-\mathrm{PS}_{27}$ block copolymer solution at various wavelengths. Inset shows the change in scattered intensity as a function of temperature measured by DLS. Arrows represent the LCST and CMT.

turbidity is expected to change the optical properties of the polymer such as its transmittance and refractive index, due to the increased Rayleigh scattering arising from the larger refractive index contrast with the solvent. Further it has been reported that PNIPAM block can self-assemble into micellar aggregates in aqueous solution at elevated temperatures [42, 43]. The PNIPAM chains are easily solvated in water and thus the block copolymer can self-assemble into core-shell micelles. We also verified the influence of temperature on the micelle formation in a dilute solution $(0.001 \mathrm{wt} \%)$ by monitoring the optical transmittance as a function of temperature for a series of wavelengths. From Figure 4, it can be seen that, apart from the dip in transmittance at the LCST a significant decrease in the transmittance is observed above $34^{\circ} \mathrm{C}$, which is identified as the critical micellization temperature (CMT).Similar increase in the scattered light intensity is noted in the $0.001 \mathrm{wt} \%$ solution in DLS as a function of temperature (inset in Figure 4). Zhou et al. [44] also observed that scattered light intensity of $0.2 \mathrm{~g} / \mathrm{L} \mathrm{PNIPAM}_{67}-b-\mathrm{PS}_{27}$ aqueous solution increases at $42{ }^{\circ} \mathrm{C}$, whereas the CMT determined by optical transmittance was $38.5^{\circ} \mathrm{C}$ and the difference was attributed to the polymer concentration effects. Since, we carried out scattered intensity and transmission measurements on the same polymer concentration, we could observe both the increase in scattered light intensity and decrease in optical transmittance at $34^{\circ} \mathrm{C}$.A look at the inset in Figure 4 reveals that at slightly elevated temperatures beyond the 
CMT, the scattered intensity saturates. The saturation in micellar size is also noted in Figure 3. Saturation in micelle size implies that no more association of the micelles occurs and they are stable. Stable micelles are formed because the investigations are carried out at very low polymer concentrations and even at these concentrations, despite the small number of ionic end-groups, the charges in the PNIPAM chains of the micelles screens the inter-micellar interactions and thus the block copolymer solution gets charge stabilization even at temperatures beyond the LCST. The refractive index of $0.005 \mathrm{wt} \%$ PNIPAM $_{67}-b$ $\mathrm{PS}_{27}$ solution increases from 1.336 to 1.352 when the temperature is varied from 20 to $40^{\circ} \mathrm{C}$ (Figure 5). In Figure 5, two regions can be identified, region (a) where there is no significant change in refractive index with increase in temperature and in region (b) refractive index increases with temperature. The refractive index values are arrived based on the number density of electronic dipoles present in the solution. For solutions which undergo phase separation there will be significant changes in bond polarization [45]. In region (a) with increase in temperature, there is not much change in the refractive index; this is because the polymer-solvent exist as a homogeneous single phase. This may not be the case when the polymer undergoes LCST as the polymer starts to phase separate in the solvent (water). It is visible from the plot that with increase in temperature a change in slope is observed at $31.6^{\circ} \mathrm{C}$, this change correlates to the LCST of the polymer determined from DLS. The gradual proximity of the hydrophobic isopropyl groups of the PNIPAM chain coupled with the chains of polystyrene results in the increase in refractive index even after the collapse of the polymer which is the region (b). From the size information obtained from DLS, not much change in the diameter is noted beyond $34^{\circ} \mathrm{C}$, thus one assumes that almost all the bound/structured water is expelled from the polymer chain, however the observation of increase in refractive index implies that the proximal hydrophobic isopropyl and polystyrene chains in the collapsed (phase separated) polymer cycles to a stable rearrangement to overcome the steric hindrance effects offered by the hydrophobic chains.

In the PNIPAM ${ }_{67}-b-\mathrm{PS}_{27}$ micelles the hydrophilic PNIPAM chains extend into water and PS forms the micelle core. The interplay of steric hindrance offered by the collapsed PNIPAM shell and the unchanged

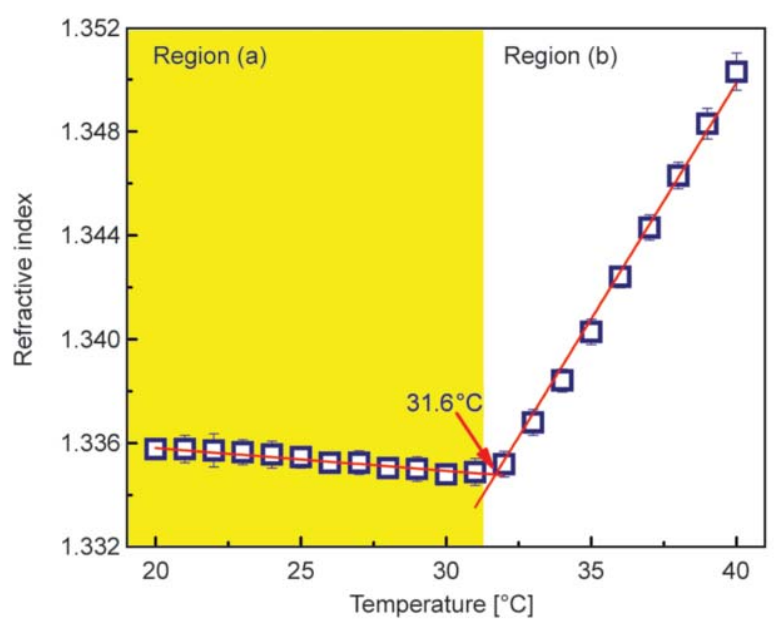

Figure 5. Refractive index as a function of temperature for 0.005 wt $\%$ PNIPAM $_{67}-b-$ PS $_{27}$ blocks copolymer solution. Intercept of the slopes corresponds to the LCST.

PS core increases the density of the electron cloud leading to an increase in the refractive index.

\subsection{Rheological characterization of PNIPAM $_{67}-b-$ PS $_{27}$ solution}

\subsubsection{Effect of temperature on the rheological behavior}

Oscillatory shear measurements on aqueous PNIPAM $_{67-} b-\mathrm{PS}_{27}$ solution has been performed as a function of temperature for micelle pre-formed 0.05 and $2 \mathrm{wt} \% \mathrm{PNIPAM}_{67}-b-\mathrm{PS}_{27}$ aqueous solution at a constant angular frequency of $\omega=3 \mathrm{rad} / \mathrm{s}$ and is shown in Figure 6. The PNIPAM ${ }_{67}-b-\mathrm{P}_{\mathrm{S} 27}$ readily forms viscoelastic solutions even at very low concentrations due to the associative nature of the hydrophilic PNIPAM segments (below LCST) and hydrophobic PS. Both the solutions follow the same trend and exhibits an apparent increase in both $G^{\prime}$ and $G^{\prime \prime}$ beyond $30^{\circ} \mathrm{C}$ with the steepness of the slope increasing at the LCST of the polymer. Below $30^{\circ} \mathrm{C}, G^{\prime}>G^{\prime \prime}$ implying the behavior of the polymer with dominant elastic response. For the 0.05 and $2 \mathrm{wt} \%$ polymer solution $G^{\prime}$ crossover $G^{\prime \prime}$ at 30.7 and $29.9^{\circ} \mathrm{C}$ respectively (inset in Figure 6) beyond which $G^{\prime \prime}>G^{\prime}$. The temperature at which $G^{\prime}$ (elastic modulus) curve intersects that of $G^{\prime \prime}$ (viscous modulus) indicates the gelsol transition point. The study proves that cross-over temperature is concentration dependent.

In general, a gel exhibits $G^{\prime}>G^{\prime \prime}$ throughout the frequency range and behaves like solid, with frequency independent of the modulus. For very dilute solutions $G^{\prime \prime}>G^{\prime}$ at low frequencies. The relaxation times 


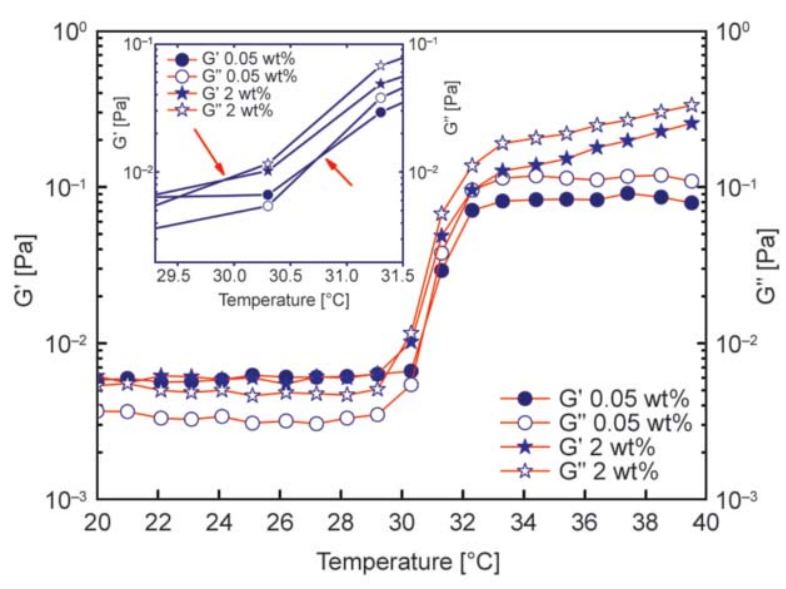

Figure 6. Storage modulus $G^{\prime}$ (solid symbols) and loss modulus $G^{\prime \prime}$ (open symbols) as a function of temperature for 0.05 and $2 \mathrm{wt} \%$ PNIPAM $_{67}-b-\mathrm{PS}_{27}$ aqueous solution. Inset shows the shift in crossover temperature with increase in concentration.

exhibit concentration dependence in which the high concentration solutions can undergo entanglements and the entanglements persist during the oscillation with mechanical behavior resembling like a solid with $G^{\prime}>G^{\prime \prime}$, whereas in the case of dilute solutions the entanglements can disentangle and flow during shear leading to $G^{\prime \prime}>G^{\prime}$. Then the solution behaves like a viscoelastic liquid. In the polymer concentrations explored in our study, the solution was flowing yet manifested solid like behavior with $G^{\prime}>G^{\prime \prime}$ and the modulus was found to be frequency dependent, with magnitude of $G^{\prime}$ two-fold higher than $G^{\prime \prime}$. This implies a weak gel behavior in the solutions explored. Weak gel behavior is previously reported in aqueous dispersion of $\kappa$-carragennan helices [46, 47], ionic liquids [48], etc. Ikeda and Nishinari [46] observed the predominance of storage modulus $G^{\prime}$ over the loss modulus $G^{\prime \prime}$ in non-aggregated $\kappa$-carrageenan helices (polysaccharide) at $20^{\circ} \mathrm{C}$ in the frequency range of $0.5-100 \mathrm{rad} / \mathrm{s}$ and $G^{\prime \prime} / G^{\prime}>0.1$, which is typical of weak gels. The weak gel-type behavior of the $\kappa$-carrageenan dispersions arises from the sufficiently long relaxation time of the topological entanglements among double-helical conformers. Thermoreversible viscoelastic to weak gel transition in ionic liquid is observed in a micellar solution of 1butyl-3-methylimidazolium chloride (BmimCl) containing $4.45 \mathrm{wt} \%$ sodium dodecylsulfate (SDS) upon the addition of lithium chloride by Saravanakumar et al. [48]. Upon heating the ionic liquid mixture $>80^{\circ} \mathrm{C}$ weak gel behavior is noted, however upon cooling, the mixture exhibited a viscoelastic liquid state. Here, the authors attribute the weak gel behavior

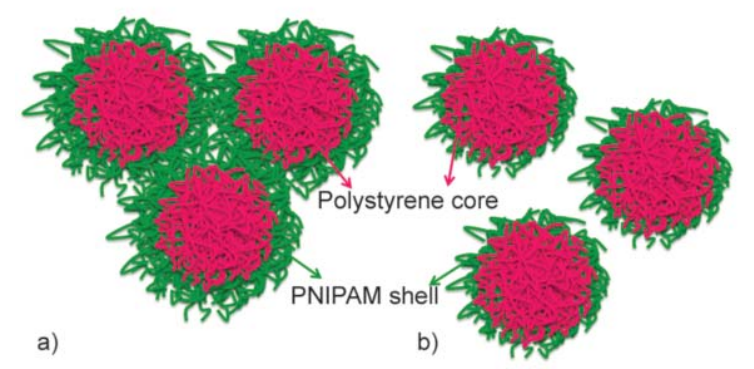

Figure 7. Schematic of the PNIPAM $67-b-\mathrm{PS}_{27}$ micelles (a) entangled before and (b) disentangled after LCST

to arise from micellar network structures in the ionic liquid mixture.

In our investigations, we observed weak gel behavior at lower temperatures and viscoelastic liquid behavior upon heating the weak gel solution. This behavior can be explained as follows;

Below the LCST the shell of PNIPAM chains in the micelles are swollen in water and form inter-micellar entanglements and thereby the swollen micelles occupy more space in the given volume and hence the solution behaves like a gel and thus $G^{\prime}>G^{\prime \prime}$, however upon crossing the LCST, the shell shrinks leading to disentanglement of the PNIPAM chains and the micelles have enough space to execute free motion as compared to their swollen state. In this state the polymer behaves like a liquid and hence $G^{\prime \prime}>G^{\prime}$. The difference in the crossover temperature for the 0.05 and $2 \mathrm{wt} \%$ polymer solution is attributed to the concentration effect. Schematic of the above explanation is shown in Figure 7.

\subsubsection{Effect of polymer concentration on the rheological behavior}

The effect of concentration of four different aqueous solutions $(0.05,0.5,1$ and $2 \mathrm{wt} \%)$ of PNIPAM $67-b$ $\mathrm{PS}_{27}$ has been carried out as a function of angular frequency at $25^{\circ} \mathrm{C}$ and is shown in Figure 8. The plot shows $G^{\prime}>G^{\prime \prime}$, for all the four concentrations with a concentration dependent behavior throughout the frequency range. Further the value of the modulii increased with increase in polymer content. The associative nature of the PNIPAM and PS segments leading to the formation of micelles causes the samples to exhibit viscoelastic properties even at a polymer concentration of $0.05 \mathrm{wt} \%$. We observed power law dependence of the modulii on the frequency with $G^{\prime} \approx \omega 2$ and $G^{\prime \prime} \approx \omega 1$ and the power law holds true in solutions where inter-micellar entanglements are present. All the four concentrations explored in the study manifested weak-gel behavior at $25^{\circ} \mathrm{C}$. 


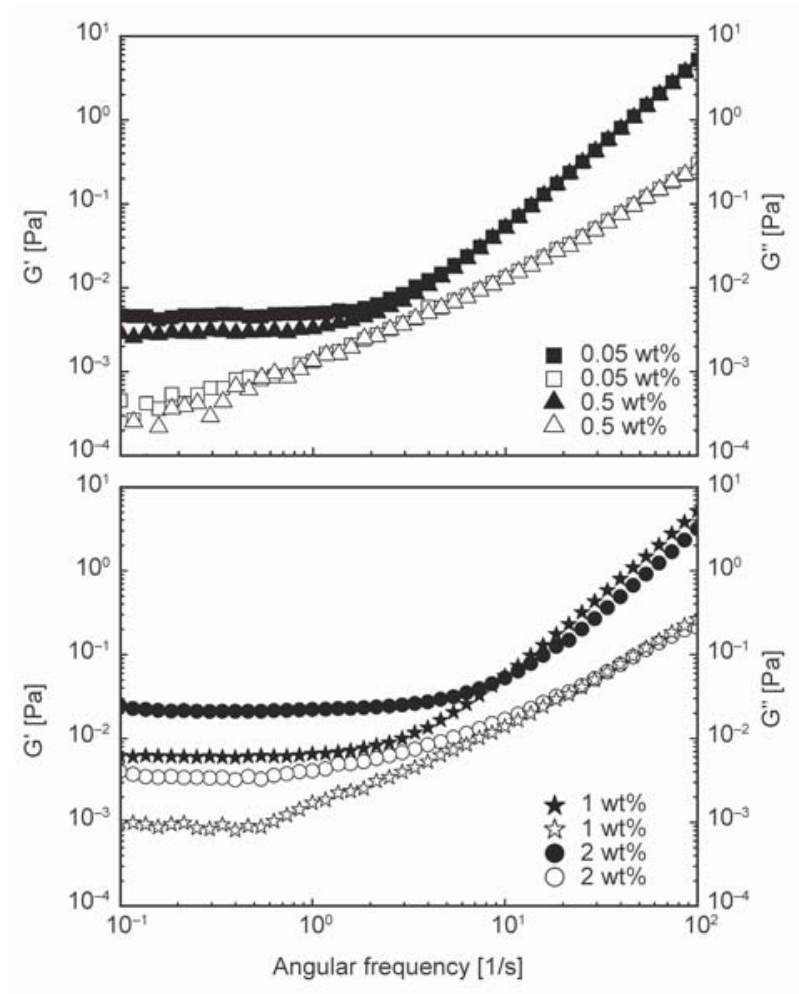

Figure 8. Storage modulus $G^{\prime}$ (solid symbols) and loss modulus $G^{\prime \prime}$ (open symbols) as a function of angular frequency for four different PNIPAM $_{67}-b-\mathrm{PS}_{27}$ concentrations $(0.05,0.5,1$ and $2 \mathrm{wt} \%)$ performed at $25^{\circ} \mathrm{C}$. The graph has been split for visualization of the data points

Figure 9 shows the plots of $G^{\prime}$ and $G^{\prime \prime}$ as function of $\omega$ at four different temperatures for the $2 \mathrm{wt} \%$ solution. At $24^{\circ} \mathrm{C} G^{\prime}>G^{\prime \prime}$ in the entire $\omega$ range, and the damping factor, $\delta\left(\delta=G^{\prime \prime} / G^{\prime}\right)$ throughout the frequency sweep ranged between $\sim 0.117-0.29$. A relatively large frequency dependence of the modulii, accompanied by a $\tan \delta$ value $>0.1$, is the signature of a weak gel [41]. The above observation thus, implies that the PNIPAM $67-b-\mathrm{PS}_{27}$ solution behaves like a weak gel at $24^{\circ} \mathrm{C}$. At $30^{\circ} \mathrm{C}$, crossover of the modulus is observed at $\omega=7.36 \mathrm{~s}^{-1}$. At $32{ }^{\circ} \mathrm{C}$ the crossover was found to be shifted to a higher $\omega=$ $25.1 \mathrm{~s}^{-1}$. Beyond $36^{\circ} \mathrm{C} G^{\prime \prime}$ dominates $G^{\prime}$ throughout the frequency range swept. This implies that the entangled chains of PNIPAM in the micelle start to recede upon crossing the LCST and temperature disentangles the polymer chains and the solution behaves like a viscoelastic liquid.

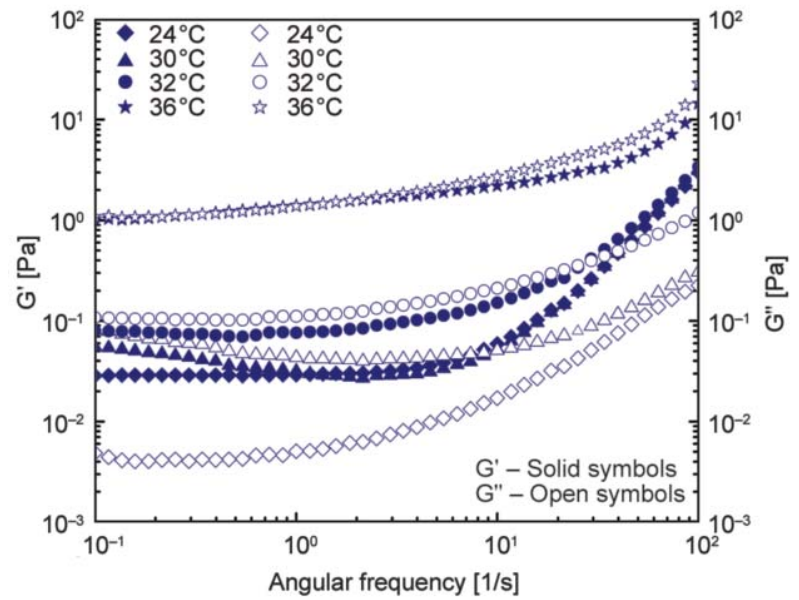

Figure 9. Storage modulus $G^{\prime}$ (solid symbols) and loss modulus $G^{\prime \prime}$ (open symbols) as a function of angular frequency in $2 \mathrm{wt} \%$ PNIPAM $_{67}-b-\mathrm{PS}_{27}$ block copolymer solution with increasing temperature

\section{Conclusions}

Water dispersible block copolymer composed of low molecular weight poly( $N$-isopropylacrylamide)- $b$ polystyrene has been synthesized by RAFT polymerization. From GPC measurements the molecular weight of PNIPAM ${ }_{67}-b-\mathrm{PS}_{27}$ block copolymer is found to be $9695 \mathrm{~g} / \mathrm{mol}$ with PDI of 1.27 . The critical micelle concentration is identified as $0.005 \mathrm{wt} \%$. The effect of temperature on the hydrodynamic diameter of the micelles formed in $0.005 \mathrm{wt} \%$ polymer solution is studied by means of DLS. From DLS, the hydrodynamic diameter of the micelles is found to be $85.2 \pm 5.66 \mathrm{~nm}$ at $25^{\circ} \mathrm{C}$. A dilute solution $(0.001 \mathrm{wt} \%)$ where micelles are absent at $25^{\circ} \mathrm{C}$ formed micelles beyond the LCST of the polymer which indicates that micelle formation also depends on the temperature and the critical micelle temperature is identified as $34^{\circ} \mathrm{C}$ by transmission spectroscopy. Rheological studies on the block copolymer solution showed that the solution exhibits weak gel-like behavior and transforms to liquid like behavior with changes in temperature, the transition point is found to be dependent on the block copolymer concentration. Unlike other reported works on sol-weak gel behaviour upon increasing the temperature in this study we observed weak gel behavior at lower temperatures and viscoelastic liquid behavior upon heating the weak gel solution. The observed weak gel to liquid behavior is due to the inter-micellar entanglement-disentanglements influenced by temperature. 


\section{Acknowledgements}

Authors acknowledge the Department of Science and Technology, New Delhi, Govt. of. India, Grant No.SR/FTP/PS046/2012, for supporting this work. Authors are thankful to Prof. Abhijit P. Deshpande and Ms. K. A. Ramya, Department of Chemical Engineering, IIT Madras for their timely help in rheological measurements.

\section{References}

[1] Ramanathan M., Tseng Y-C., Ariga K., Darling S. B.: Emerging trends in metal-containing block copolymers: Synthesis, self-assembly, and nanomanufacturing applications. Journal of Materials Chemistry C, 1, 2080 2091 (2013). https://doi.org/10.1039/c3tc00930k

[2] Marencic A. P., Register R. A.: Controlling order in block copolymer thin films for nanopatterning applications. Annual Review of Chemical and Biomolecular Engineering, 1, 277-297 (2010).

https://doi.org/10.1146/annurev-chembioeng-073009-101007

[3] Chen H-C., Su Y-W., Wei K-H.: A block copolymer enhances the efficiency of small-molecule bulk-heterojunction photovoltaics. Journal of Materials Chemistry A, 4, 2228-2235 (2016).

https://doi.org/10.1039/C5TA08932H

[4] Guo C., Lin Y-H., Witman M. D., Smith K. A., Wang C., Hexemer A., Strzalka J., Gomez E. D., Verduzco R.: Conjugated block copolymer photovoltaics with near 3\% efficiency through microphase separation. Nano Letters, 13, 2957-2963 (2013). https://doi.org/10.1021/nl401420s

[5] Ge Z., Liu S.: Functional block copolymer assemblies responsive to tumor and intracellular microenvironments for site-specific drug delivery and enhanced imaging performance. Chemical Society Reviews, 42, 7289-7325 (2013).

https://doi.org/10.1039/c3cs60048c

[6] Rösler A., Vandermeulen G. W., Klok H-A.: Advanced drug delivery devices via self-assembly of amphiphilic block copolymers. Advanced Drug Delivery Reviews, 64, 270-279 (2012).

https://doi.org/10.1016/j.addr.2012.09.026

[7] Kang Y., Walish J. J., Gorishnyy T., Thomas E. L.: Broadwavelength-range chemically tunable block-copolymer photonic gels. Nature Materials, 6, 957-960 (2007). https://doi.org/10.1038/nmat2032

[8] Urbas A. M., Maldovan M., DeRege P., Thomas E. L.: Bicontinuous cubic block copolymer photonic crystals. Advanced Materials, 14, 1850-1853 (2002). https://doi.org/10.1002/adma.200290018

[9] Kang C., Kim E., Baek H., Hwang K., Kwak D., Kang Y., Thomas E. L.: Full color stop bands in hybrid organic/inorganic block copolymer photonic gels by swelling-freezing. Journal American Chemical Society, 131, 7538-7539 (2009).

https://doi.org/10.1021/ja9021478
[10] Deng T., Chen C., Honeker C., Thomas E. L.: Two-dimensional block copolymer photonic crystals. Polymer, 44, 6549-6553 (2003). https://doi.org/10.1016/j.polymer.2003.07.002

[11] Lu Y., Xia H., Zhang G., Wu C.: Electrically tunable block copolymer photonic crystals with a full color display. Journal of Materials Chemistry, 19, 5952-5955 (2009). https://doi.org/10.1039/b905760a

[12] Song D-P., Li C., Li W., Watkins J. J.: Block copolymer nanocomposites with high refractive index contrast for one-step photonics. ACS Nano, 10, 1216-1223 (2016). https://doi.org/10.1021/acsnano.5b06525

[13] Mayadunne R. T., Rizzardo E., Chiefari J., Krstina J., Moad G., Postma A., Thang S. H.: Living polymers by the use of trithiocarbonates as reversible addition-fragmentation chain transfer (RAFT) agents: ABA triblock copolymers by radical polymerization in two steps. Macromolecules, 33, 243-245 (2000).

https://doi.org/10.1021/ma991451a

[14] Harrisson S., Wooley K. L.: Shell-crosslinked nanostructures from amphiphilic AB and ABA block copolymers of styrene-alt-(maleic anhydride) and styrene: Polymerization, assembly and stabilization in one pot. Chemical Communications, 2005, 3259-3261 (2005). https://doi.org/10.1039/B504313A

[15] Zeng J., Shi K., Zhang Y., Sun X., Deng L., Guo X., Du Z., Zhang B.: Synthesis of poly( $N$-isopropylacrylamide)b-poly(2-vinylpyridine) block copolymers via RAFT polymerization and micellization behavior in aqueous solution. Journal of Colloid and Interface Science, 322, 654-659 (2008).

https://doi.org/10.1016/j.jcis.2008.03.032

[16] Turner S. R., Blevins R. W.: Photoinitiated block copolymer formation using dithiocarbamate free radical chemistry. Macromolecules, 23, 1856-1859 (1990).

https://doi.org/10.1021/ma00208a050

[17] Lambrinos P., Tardi M., Polton A., Sigwalt P.: The mechanism of the polymerization of n.butyl acrylate initiated with $N, N$-diethyl dithiocarbamate derivatives. European Polymer Journal, 26, 1125-1135 (1990).

https://doi.org/10.1016/0014-3057(90)90014-U

[18] Lai J. T., Shea R.: Controlled radical polymerization by carboxyl- and hydroxyl-terminated dithiocarbamates and xanthates. Journal of Polymer Science Part A: Polymer Chemistry, 44, 4298-4316 (2006).

https://doi.org/10.1002/pola.21532

[19] Shi P-J., Li Y-G., Pan C-Y.: Block and star block copolymers by mechanism transformation $\mathrm{X}$. Synthesis of poly(ethylene oxide) methyl ether/polystyrene/poly (L-lactide) ABC miktoarm star copolymers by combination of RAFT and ROP. European Polymer Journal, 40, 1283-1290 (2004).

https://doi.org/10.1016/j.eurpolymj.2004.02.024 
[20] Quinn J. F., Chaplin R. P., Davis T. P.: Facile synthesis of comb, star, and graft polymers via reversible addition-fragmentation chain transfer (RAFT) polymerization. Journal of Polymer Science Part A: Polymer Chemistry, 40, 2956-2966 (2002).

https://doi.org/10.1002/pola.10369

[21] Barner-Kowollik C., Buback M., Charleux B., Coote M. L., Drache M., Fukuda T., Goto A., Klumperman B., Lowe A. B., Mcleary J. B., Moad G., Monteiro M. J., Sanderson R. D., Tonge M. P., Vana P.: Mechanism and kinetics of dithiobenzoate-mediated RAFT polymerization. I. The current situation. Journal of Polymer Science Part A: Polymer Chemistry, 44, 5809-5831 (2006). https://doi.org/10.1002/pola.21589

[22] Zheng Q., Pan C-Y.: Preparation and characterization of dendrimer-star PNIPAAM using dithiobenzoate-terminated PPI dendrimer via RAFT polymerization. European Polymer Journal, 42, 807-814 (2006).

https://doi.org/10.1016/j.eurpolymj.2005.10.002

[23] Zhang Y. L., Dou X. W., Jin T.: Synthesis and self-assembly behavior of amphiphilic diblock copolymer dextran-block-poly( $\varepsilon$-caprolactone) (DEX- $b$-PCL) in aqueous media. Express Polymer Letters, 4, 599-610 (2010). https://doi.org/10.3144/expresspolymlett.2010.75

[24] Tang Y. C., Wu J. H., Duan J. X.: The micellization and dissociation transitions of thermo-, $\mathrm{pH}$ - and sugar-sensitive block copolymer investigated by laser light scattering. Express Polymer Letters, 8, 647-656 (2012). https://doi.org/10.3144/expresspolymlett.2012.69

[25] Poggi E., Guerlain C., Debuigne A., Detrembleur C., Gigmes D., Hoeppener S., Schubert U. S., Fustin C-A., Gohy J-F.: Stimuli-responsive behavior of micelles prepared from a poly(vinyl alcohol)-block-poly(acrylic acid)-block-poly(4-vinylpyridine) triblock terpolymer. European Polymer Journal, 62, 418-425 (2015).

http://dx.doi.org/10.1016/j.eurpolymj.2014.06.026

[26] Brijitta J., Tata B. V. R., Joshi R. G., Kaliyappan T.: Random HCP and FCC structures in thermoresponsive microgel crystals. The Journal of Chemical Physics, 131, 074904/1-074904/5 (2009).

https://doi.org/10.1063/1.3210765

[27] Brijitta J., Tata B. V. R., Kaliyappan T.: Phase behavior of poly( $N$-isopropylacrylamide) nanogel dispersions: Temperature dependent particle size and interactions. Journal of Nanoscience and Nanotechnology, 9, 5323 5328 (2009).

https://doi.org/10.1166/jnn.2009.1144

[28] Brijitta J., Tata B.V. R., Joshi R. G.: Controlled synthesis of monodisperse thermo-responsive nanogel particles. Journal of Polymer Research, 22, 36/1-36/8 (2015). https://doi.org/10.1007/s10965-015-0674-x

[29] Joshi R. G., Tata B. V. R., Brijitta J.: Dynamics in thermo-responsive nanogel crystals undergoing melting. The Journal of Chemical Physics, 139, 124901/112490/8 (2013).

https://doi.org/10.1063/1.4821584
[30] Tata B. V. R., Brijitta J., Joshi R. G.: Thermo-responsive nanogel dispersions: Dynamics and phase behaviour. International Journal of Advances in Engineering Sciences and Applied Mathematics, 5, 240-249 (2011). https://doi.org/10.1007/s12572-010-0016-5

[31] Lee S. J., Han B. R., Park S. Y., Han D. K., Kim S. C.: Sol-gel transition behavior of biodegradable three-arm and four-arm star-shaped PLGA-PEG block copolymer aqueous solution. Journal of Polymer Science Part A: Polymer Chemistry, 44, 888-899 (2006). https://doi.org/10.1002/pola.21193

[32] Jeong B., Kim S. W., Bae Y. H.: Thermosensitive solgel reversible hydrogels. Advanced Drug Delivery Reviews, 64, 154-162 (2012). http://dx.doi.org/10.1016/j.addr.2012.09.012

[33] Chung Y. M., Simmons K. L., Gutowska A., Jeong B.: Sol-gel transition temperature of PLGA-g-PEG aqueous solutions. Biomacromolecules, 3, 511-516 (2002). https://doi.org/10.1021/bm0156431

[34] Convertine A. J., Lokitz B. S., Vasileva Y., Myrick L. J., Scales C. W., Lowe A. B., McCormick C. L.: Direct synthesis of thermally responsive DMA/NIPAM diblock and DMA/NIPAM/DMA triblock copolymers via aqueous, room temperature RAFT polymerization. Macromolecules, 39, 1724-1730 (2006).

https://doi.org/10.1021/ma0523419

[35] Davis K. A., Paik H-J., Matyjaszewski K.: Kinetic investigation of the atom transfer radical polymerization of methyl acrylate. Marcomolecules, 32, 1767-1776 (1999). https://doi.org/10.1021/ma9815051

[36] Moineau G., Minet M., Dubois P., Teyssié P., Senninger T., Jérôme R.: Controlled radical polymerization of (meth)acrylates by ATRP with $\mathrm{NiBr}_{2}\left(\mathrm{PPh}_{3}\right)_{2}$ as catalyst. Marcomolecules, 32, 27-35 (1999). https://doi.org/10.1021/ma980995u

[37] Monteiro M. J., Hodgson M., De Brouwer H.: The influence of RAFT on the rates and molecular weight distributions of styrene in seeded emulsion polymerizations. Journal of Polymer Science Part A: Polymer Chemistry, 38, 3864-3874 (2000).

https://doi.org/10.1002/10990518(20001101)38:21<3864::AID-POLA30>3.0.CO;2-3

[38] Cui S., Pang X., Zhang S., Yu Y., Ma H., Zhang.: Unexpected temperature-dependent single chain mechanics of poly ( $N$-isopropyl-acrylamide) in water. Langmuir, 28, 5151-5157 (2012). https://doi.org/10.1021/la300135w

[39] Lutz J-F., Akdemir Ö., Hoth A.: Point by point comparison of two thermosensitive polymers exhibiting a similar LCST: Is the age of poly(NIPAM) over? Journal of the American Chemical Society, 128, 13046-13047 (2006).

https://doi.org/10.1021/ja065324n

[40] Debord J. D., Lyon L. A.: Synthesis and characterization of $\mathrm{pH}$-responsive copolymer microgels with tunable volume phase transition temperatures. Langmuir, 19, 7662-7664 (2003).

https://doi.org/10.1021/la0342924 
[41] Berndt I., Richtering W.: Doubly temperature sensitive core-shell microgels. Marcromolecules, 36, 8780-8785 (2003).

https://doi.org/10.1021/ma034771

[42] Papagiannopoulos A., Zhao J., Zhang G., Pispas S., Radulescu A.: Thermoresponsive aggregation of PS-PNIPAM-PS triblock copolymer: A combined study of light scattering and small angle neutron scattering. European Polymer Journal, 56, 59-68 (2014). https://doi.org/10.1016/j.eurpolymj.2014.04.013

[43] Luo Y-L., Yu W., Xu F., Zhang L-L.: Novel thermo-responsive self-assembly micelles from a double brushshaped PNIPAM- $g$-(PA- $b$-PEG- $b$-PA)- $g$-PNIPAM block copolymer with PNIPAM polymers as side chains. Journal of Polymer Science Part A: Polymer Chemistry, 50, 2053-2067 (2012).

https://doi.org/10.1002/pola.25980

[44] Zhou X., Ye X., Zhang G.: Thermoresponsive triblock copolymer aggregates investigated by laser light scattering. The Journal of Physcial Chemistry-B, 111, 51115115 (2007).

https://doi.org/10.1021/jp0659352
[45] Philipp M., Aleksandrova R., Müller U., Ostermeyer M., Sanctuary R., Müller-Buschbaum P., Krüger J. K.: Molecular versus macroscopic perspective on the demixing transition of aqueous PNIPAM solutions by studying the dual character of the refractive index. Soft Matter, 10, 7297-7305 (2014).

https://doi.org/10.1039/C4SM01222D

[46] Ikeda S., Nishinari K.: 'Weak gel'-type rheological properties of aqueous dispersions of nonaggregated $\kappa$-carrageenan helices. Journal of Agricultural and Food Chemistry, 49, 4436-4441 (2001). https://doi.org/10.1021/jf0103065

[47] Rodd A. B., Davis C. R., Dunstan D. E., Forrest B. A., Boger D. V.: Rheological characterisation of 'weak gel' carrageenan stabilised milks. Food Hydrocolloids, 14, 445-454 (2000). https://doi.org/10.1016/S0268-005X(00)00024-2

[48] Saravanakumar K., Tata B. V. R., Aswal V. K.: Thermoreversible viscoelastic to weak gel transition in a micellar ionic liquid with salt. Colloids and Surfaces A: Physicochemical and Engineering Aspects, 414, 359365 (2012).

https://doi.org/10.1016/j.colsurfa.2012.08.061 